OPEN ACCESS

Edited by: El-Sayed Salama, Lanzhou University, China

Reviewed by: Nadia Samak,

Institute of Process Engineering

(CAS), China

Moonis Ali Khan,

King Saud University, Saudi Arabia

*Correspondence:

Christiane Funk

christiane.funk@umu.se

Specialty section:

This article was submitted to Marine Biotechnology,

a section of the journal

Frontiers in Marine Science

Received: 07 December 2021

Accepted: 25 January 2022

Published: 08 March 2022

Citation:

Plöhn M, Scherer K, Stagge S,

Jönsson LJ and Funk C (2022)

Utilization of Different Carbon Sources by Nordic Microalgae Grown Under

Mixotrophic Conditions.

Front. Mar. Sci. 9:830800.

doi: $10.3389 /$ fmars.2022.830800

\section{Utilization of Different Carbon Sources by Nordic Microalgae Grown Under Mixotrophic Conditions}

\author{
Martin Plöhn, Kim Scherer, Stefan Stagge, Leif J. Jönsson and Christiane Funk* \\ Department of Chemistry, Umeå University, Umeå, Sweden
}

Microalgae are promising candidates for sustainable wastewater treatment coupled to the production of biofuel, bioplastic and/or bio-fertilizers. In Nordic countries, however, light is a limiting factor for photosynthesis and biomass production during the winter season. Compared to municipal wastewater, industrial wastewater streams from the pulp and paper industry contain lower amounts of nitrogen, but high concentrations of carbon sources, which could be utilized by microalgae to enhance biomass production in limiting light. This study focused on the utilization of methanol, glycerol and xylose by five different Nordic microalgae [Chlorella vulgaris (13-1), Coelastrella sp. (3-4), Desmodesmus sp. (2-6), Chlorococcum sp. (MC1) and Scotiellopsis reticulata (UFA2)] grown under mixotrophic conditions. Two of these strains, i.e., Chlorococcum sp. (MC1) and Scotiellopsis reticulata (UFA-2) were able to grow in the presence of xylose or methanol at concentrations of $6 \mathrm{~g} \mathrm{~L}^{-1}$, or $3 \%$, respectively, in a 12/12 $\mathrm{h}$ day/night cycle. HPLC analysis confirmed the consumption of those substrates. Glycerol $(2.3 \mathrm{~g}$ $\mathrm{L}^{-1}$ ) was tolerated by all strains and increased growth for Chlorella vulgaris (131), while higher concentrations (20 $\mathrm{g} \mathrm{L}^{-1}$ ) were only tolerated by Chlorococcum sp. (MC-1). Fourier-transform infrared spectroscopy, performed after growth in presence of the dedicated carbon source, indicated an increase in the fingerprint region of the carbohydrate fraction. This was particularly the case for Chlorococcum sp. (MC1), when grown in presence of glycerol, and Scotiellopsis reticulata (UFA-2), when grown in presence of xylose. Therefore, these strains could be potential candidates for the production of biofuels, e.g., bioethanol or biogas. We could show that Nordic microalgae are able to grow on various carbon sources; the actual uptake rates are low during a 12/12 $\mathrm{h}$ day/night cycle requesting additional optimization of the cultivation conditions. Nonetheless, their potential to use pulp and paper wastestreams for cheap and sustainable biomass production is high and will support the development of new technologies, turning waste-streams into resources in a circular economy concept.

Keywords: Nordic microalgae, carbohydrate, wastewater treatment, glycerol, methanol, xylose 


\section{INTRODUCTION}

The annual global water demand is about $4,600 \mathrm{~km}^{3}$ according to the World Water Development Report from 2019. About 20\% $\left(\sim 920 \mathrm{~km}^{3}\right)$ of the water is used by industry other than agriculture (Boretti and Rosa, 2019). The economy of the Nordic countries is heavily dependent on wood industry and on companies using wood as raw material. The production of cellulose-based products (pulp, paper, and paperboard) generates 870 million $\mathrm{m}^{3}$ of wastewater per year just in Sweden. Although most of it is recovered and recycled roughly 37 million $\mathrm{m}^{3}$ of water remain to be cleaned (Munthe et al., 2011). These waste streams are rich in phosphorus and organic carbon, e.g., cellulose or hemicellulose, with xylose being one of the major compounds, turning them into potentially high valuable resources for microalgal growth. Also waste streams from oil crop based biofuel production contain large amounts of carbon in form of crude glycerol that might be a cheap source of carbon for microalgal cultivation (Dobson et al., 2012).

Biological wastewater remediation based on microalgae has been recognized as a low-energy and environmental-friendly alternative compared to conventional wastewater treatment processes (e.g., aerobic activated sludge, anaerobic digestion), due to its low operational costs, simple bioreactor design, low carbon footprint and high nutrient removal efficiency (Acién et al., 2016; Molinuevo-Salces et al., 2019; Goswami et al., 2021; Plöhn et al., 2021). Microalgae are highly productive photosynthetic organisms; their fast growth allows harvesting after short times. Furthermore, the aquatic growth of microorganisms in tanks or ponds requires marginal land and allows a continuous, automated system with very high flow-through capacities. They are able to utilize nutrients like nitrogen and phosphate, while also using various carbon sources besides carbon dioxide. The produced microalgal biomass then can be used to produce biofertilizers or biostimulants or it can serve as feedstock for biofuels, or bioplastics production (Gouveia, 2011; Sivaramakrishnan and Incharoensakdi, 2018; Onen Cinar et al., 2020; Mehariya et al., 2021). Microalgae also actively contribute to the removal of heavy metals, pharmaceuticals and other emerging contaminants like personal care products found in urban and/or industrial wastewaters (Suresh Kumar et al., 2015; Gojkovic et al., 2019; Hena et al., 2021; Spain et al., 2021).

Efficient microalgal growth depends on a variety of different factors (biotic and abiotic) like temperature and $\mathrm{pH}$ or the presence of algal pathogens (Christenson and Sims, 2011). The most important obstacle for microalgal cultivation in Nordic countries is the need of sunlight. However, some microalgal strains are able to grow not only photoautotrophically, but also mixotrophically or even heterotrophically. While photoautotrophic strains perform photosynthesis to convert carbon dioxide into organic matter, heterotrophic growth utilizes organic carbon in the absence of light. The combination of these two conditions is termed mixotrophic; mixotrophic growing strains can utilize different carbon sources depending on the availability of light. Several studies, partially with indigenous strains, show that mixotrophic growth can outcompete photoautotrophic or heterotrophic growth (Kong et al., 2013; Rai et al., 2013; Leite et al., 2015; Ferro et al., 2019a,b).

The aim of this study was to evaluate the ability of our Nordic microalgae to tolerate and take up carbon sources that appear in certain waste streams, i.e., from the pulp and paper industry or from biofuel production. Five different strains from three different microalgal families were grown mixotrophically in the presence of either glycerol, methanol or xylose. Microalgal growth and health were observed during the experiment, and at its end the microalgal biomass was analyzed by Diffuse Reflectance Infrared Fourier Transform Spectroscopy (DRIFTS) to understand the impact of the carbon source on the biomass composition (Gorzsás and Sundberg, 2014; Rolton et al., 2020). The Nordic microalgae were able to grow on various carbon sources; the actual uptake rates were low during a 12/12 $\mathrm{h}$ day/night cycle requesting additional optimization of the cultivation conditions. Xylose is known to inhibit microalgal growth (Yang et al., 2014); the identification of xylose-tolerant strains, which also consume xylose for their biomass production, therefore highlights the high potential of Nordic strains to turn waste streams into resources. As Nordic countries heavily depend on forest industry this knowledge will support the development of sustainable processes.

\section{MATERIALS AND METHODS}

\section{Algal Cultivation}

The Nordic strains used in this work were collected from different water bodies in Sweden (Ferro et al., 2018). Microalgal cultures were maintained on $B G 11$ agar plates at $20^{\circ} \mathrm{C}$ in continuous light with an intensity of $50 \mu \mathrm{mol} \mathrm{m}{ }^{-2} \mathrm{~s}^{-1}$. Unless otherwise noted, all reagents were purchased from Merck/Sigma Aldrich.

Five selected strains (Table 1) were grown under sterile conditions in Erlenmeyer flasks filled with $50 \mathrm{~mL}$ Bold's Basal Medium (BBM) (Bischoff and Bold, 1963), pH 7.2, and shaken at $120 \mathrm{rpm}, 17^{\circ} \mathrm{C}$ and $50 \mu \mathrm{mol} \mathrm{m}{ }^{-2} \mathrm{~s}^{-1}$ (Incubator shaker, New Brunswick) until the cultures reached mid-log phase (5-7 days). Triplicates of these pre-inoculi were diluted with BBM to a final optical density $680 \mathrm{~nm}\left(\mathrm{OD}_{680}\right)$ of $0.1 \mathrm{in} 100 \mathrm{~mL}$ Erlenmeyer flasks filled up to $30 \%$. Then mixotrophic cultivation was started by supplementing $\mathrm{BBM}$ with either $2.3 \mathrm{~g} \mathrm{~L}^{-1}$ or $20 \mathrm{~g} \mathrm{~L}^{-1}$ glycerol (VWR Chemicals, Glycerin Ph. Eur., USP, BP), 1.5\% (v/v) or 3\% $(\mathrm{v} / \mathrm{v})$ methanol (VWR Chemicals, $\geq 98.5 \%$, Technical) or $3 \mathrm{~g} \mathrm{~L}^{-1}$ or $6 \mathrm{~g} \mathrm{~L}^{-1}$ xylose (Thermo Scientific, L (-) Xylose, $\geq 99 \%$ ). Each culture was grown for 5 days in the incubator shaker illuminated at a day/night cycle of $12 \mathrm{~h}$. Microalgal growth was compared to a photoautotrophic control (BBM in the absence of external carbon source) to monitor the effect of mixotrophic growth. In addition, a control with the corresponding carbon source was incubated in the absence of microalgae to monitor photodegradation or evaporation of the carbohydrate.

To exclude bacterial contamination of the strains an aliquot $(1 \mathrm{~mL})$ of each microalgal culture was incubated in LB medium (Bertani, 1951) at $37^{\circ} \mathrm{C}$ for $24 \mathrm{~h}$ in the dark and then investigated microscopically. 
TABLE 1 | Nordic microalgal strains and their assigned strain ID.

\begin{tabular}{lccc}
\hline Family & Strain name & Strain ID & Source \\
\hline Chlorellaceae & Chlorella vulgaris & $13-1$ & Umeå (MWW) \\
Chlorococcaceae & Chlorococcum sp. & MC-1 & Bäckhammar (FW) \\
Scenedesmaceae & Coelastrella sp. & $3-4$ & Umeå (MWW) \\
Scenedesmaceae & Desmodesmus sp. & $2-6$ & Skåne-Lake Ringsjön (FW) \\
Scenedesmaceae & Scotiellopsis reticulata & UFA-2 & Dåva (MWW)
\end{tabular}

FW, fresh water; MWW, municipal wastewater (Ferro et al., 2018).

\section{Analysis of Culture Growth (Optical Density, Cell Number, and Maximum Quantum Yield of Photosystem II)}

Culture growth was determined by measuring the optical density daily at three different wavelengths $(530 \mathrm{~nm}, 680 \mathrm{~nm}$, and $750 \mathrm{~nm})\left(\mathrm{OD}_{530}, \mathrm{OD}_{680}\right.$, and $\left.\mathrm{OD}_{750}\right)$ using a T90 + UV/VIS spectrophotometer (PG Instruments Ltd.) and $10 \mathrm{~mm}$ light-path polystyrene semi-microcuvettes (Sarstedt). Cell numbers were measured every 2nd day using a Beckman Coulter Multisizer 3 (aperture size $70 \mu \mathrm{m}$, analytical volume $100 \mu \mathrm{L}$ ) and the Multisizer software (Version 3.53), and expressed in $10^{6}$ cells per mL culture (Barbosa et al., 2004).

Health of the microalgal cultures was determined by analyzing the maximum quantum yield (Qy) of Photosystem II (Rolton et al., 2020). Chlorophyll fluorescence was measured with a portable pulse-amplitude-modulation fluorimeter (Aqua Pen AP-100, Photon Systems Instruments, Czechia) after dark adaptation of the samples for $30 \mathrm{~min}$. To avoid overflow errors due to high fluorescence signals the samples were diluted to an optical density of 0.5 at $680 \mathrm{~nm}$.

\section{Determination of Substrate Concentrations}

Microalgal carbon uptake from the medium was analyzed according to Sluiter et al. (2008). Carbon sources in the samples were analyzed with a Thermo Fisher Scientific Dionex UltiMate 3000 HPLC system (Germering, Germany), which was equipped with an autosampler, an RI detector, a ternary pump, and a degasser. The separation of the samples was performed on an Aminex HPX-87H column (Bio-Rad Laboratories AB, Solna, Sweden). It was operated at $55^{\circ} \mathrm{C}$ with isocratic elution performed at a flow rate of $0.6 \mathrm{~mL} / \mathrm{min}$ using a $0.01 \mathrm{~N}$ aqueous solution of sulfuric acid as the mobile phase. External quantification was applied with high purity analytical standards. The acquired data was evaluated using Chromeleon 7.2 data management software (Thermo Fisher Scientific).

\section{Fourier Transform Infrared Spectroscopy Analysis of Microalgal Biomass}

Fourier transform infrared spectroscopy (FTIR) analyses were performed on freeze-dried microalgal biomass according to the protocol established by Gorzsás and Sundberg (2014). After sample acquisition on the last day of the time course microalgal biomass was harvested by centrifugation for $5 \mathrm{~min}$ at $3000 \mathrm{~g}$. To avoid remaining carbon source residues the harvested biomass
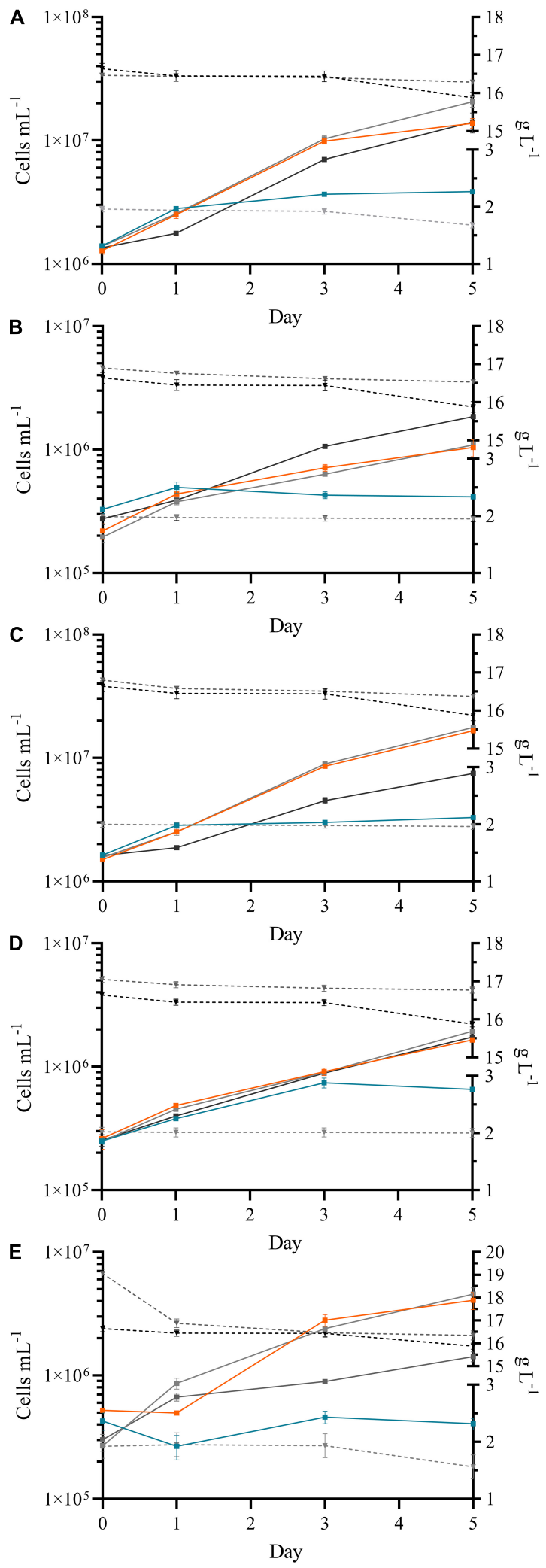

FIGURE 1 | Growth curves of Chlorella vulgaris (13-1) (A), Chlorococcum sp. (MC-1) (B), Coelastrella sp. (3-4) (C), Desmodesmus sp. (2-6) (D) and

(Continued) 
FIGURE 1 | Scotiellopsis reticulata (UFA-2) (E) under mixotrophic growth in the presence of glycerol. The presence of two different concentrations of glycerol [2.3 $\mathrm{g} \mathrm{L}^{-1}$ (light gray) and $20 \mathrm{~g} \mathrm{~L}^{-1}$ (dark gray)] were compared to a control lacking the carbon source in the medium (photoautotroph, orange) and a negative control lacking nitrogen in the medium (no algal growth, light blue). The concentration of glycerol in the medium (dashed gray line, in $\mathrm{g} / \mathrm{L}$ ) during the time course of the experiment and in the negative control lacking algae (dashed black line) are shown on the right $y$-axis.

was washed twice with Milli-Q water and centrifuged again. The biomass then was freeze-dried, mixed with $\mathrm{KBr}$ (FT-IR grade, $\geq 99 \%$ trace metal basis) and analyzed by Diffuse Reflectance Infrared Fourier Transform Spectroscopy (DRIFTS). Spectra were recorded in the range of $400-4,000 \mathrm{~cm}^{-1}$ (128 scans per sample with a spectral resolution of $4 \mathrm{~cm}^{-1}$ ) using OPUS (version 6.5). After import into MATLAB the infrared spectra were processed using a free open-source MATLAB-based script provided by the Vibrational Spectroscopy Core Facility at Umeå University. The spectra were baseline corrected by asymmetric least squares $(\lambda=10,000,000, p=0.001)$, normalized to the amide I band (Region MinMax 1,600-1,760 $\mathrm{cm}^{-1}$ ) and slightly smoothed (Savitzky-Golay smoothing, 1st order polynomial, frame $=5$ ). For further analysis of the recorded spectra the 2nd derivative was calculated.

\section{Statistical Analysis}

A Repeated Measures One-Way ANOVA with multiple comparisons and a paired, two -tailed $t$-test was performed using Prism 9.20 Software (GraphPad) to determine the differences in microalgal growth and the carbon source concentrations.

\section{RESULTS AND DISCUSSION}

\section{Growth of Nordic Microalgae in the Presence of Glycerol}

Glycerol is the main by-product in the production of biodiesel and therefore large amounts are present in the corresponding waste streams (Yang et al., 2012). The fact that glycerol is highly abundant, easily accessible and cheap makes it a very promising candidate as a carbon source for microalgae. Several studies on mixotrophic growth of microalgae using glycerol have been performed, showing varying results. Increased growth and up to 10 -fold accumulation of biomass of Chlorella vulgaris, for example, was reported in the presence of glycerol, but also growth inhibition (Liang et al., 2009; Heredia-Arroyo et al., 2011). Here five different Nordic microalgal strains were screened regarding their ability to grow in the presence of glycerol. The amount of glycerol in the growth medium over time was analyzed to investigate its uptake by the microalgal strains. All strains were able to grow in presence of glycerol at a concentration of $2.3 \mathrm{~g} \mathrm{~L}^{-1}$ (Figure 1), but only Chlorella vulgaris (13-1) showed a significantly $(p=0.049)$ better growth compared to the glycerol-free control, and the glycerol concentration in its medium decreased significantly $(p=0.001)$. This result on a Nordic C. vulgaris strain is supported by previous studies on C. vulgaris strains derived from the Netherlands and Taiwan (Liang et al., 2009; Lin and $\mathrm{Wu}, 2015)$. Interestingly, even high concentrations of glycerol $\left(20 \mathrm{~g} \mathrm{~L}^{-1}\right)$ were tolerated by the Nordic Chlorella vulgaris (13-1) (Figure 1A) with no significant difference toward the control $(p=0.718)$, while the other Chlorella vulgaris strains produced less biomass or their growth even was inhibited at high

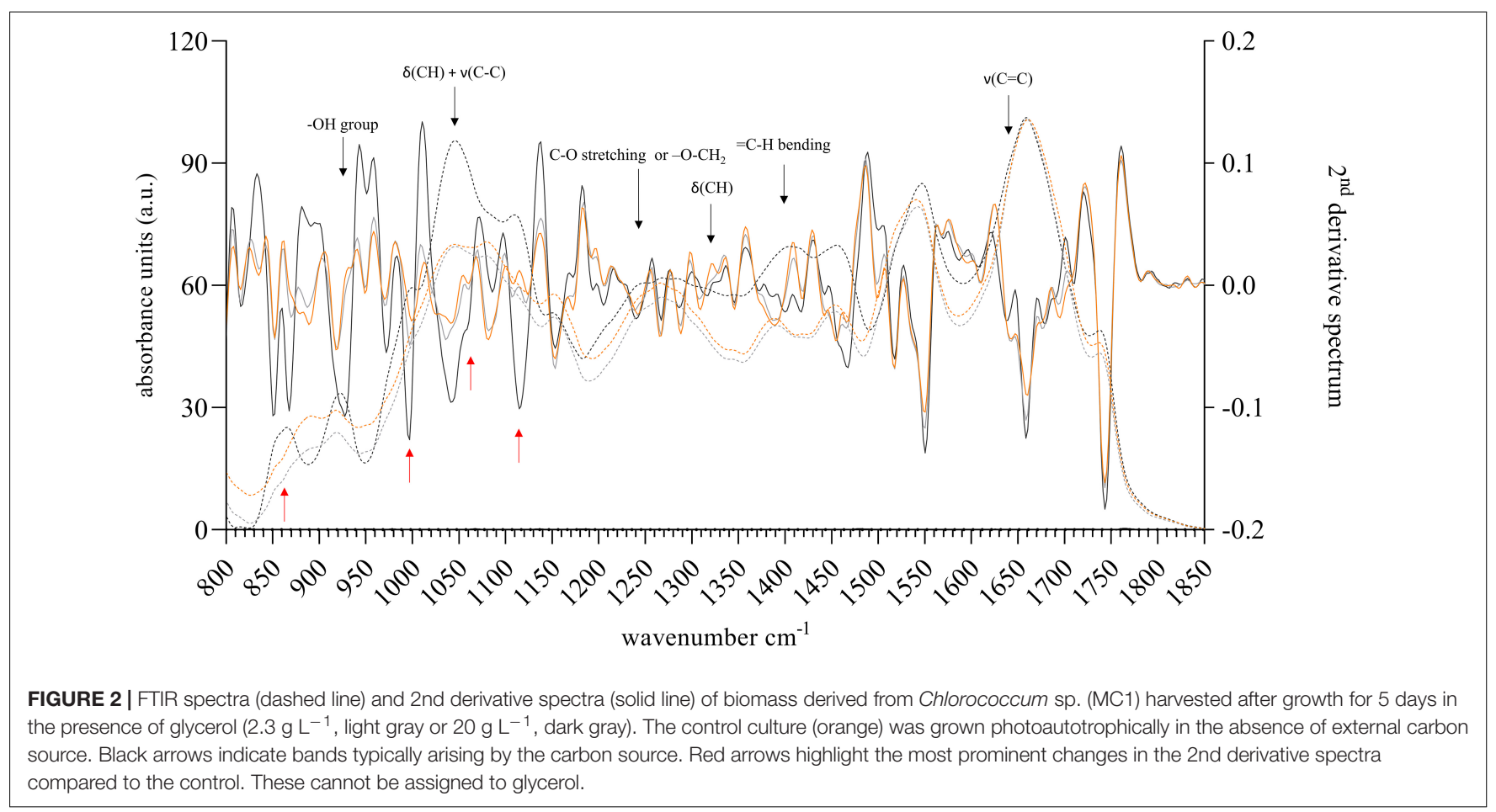



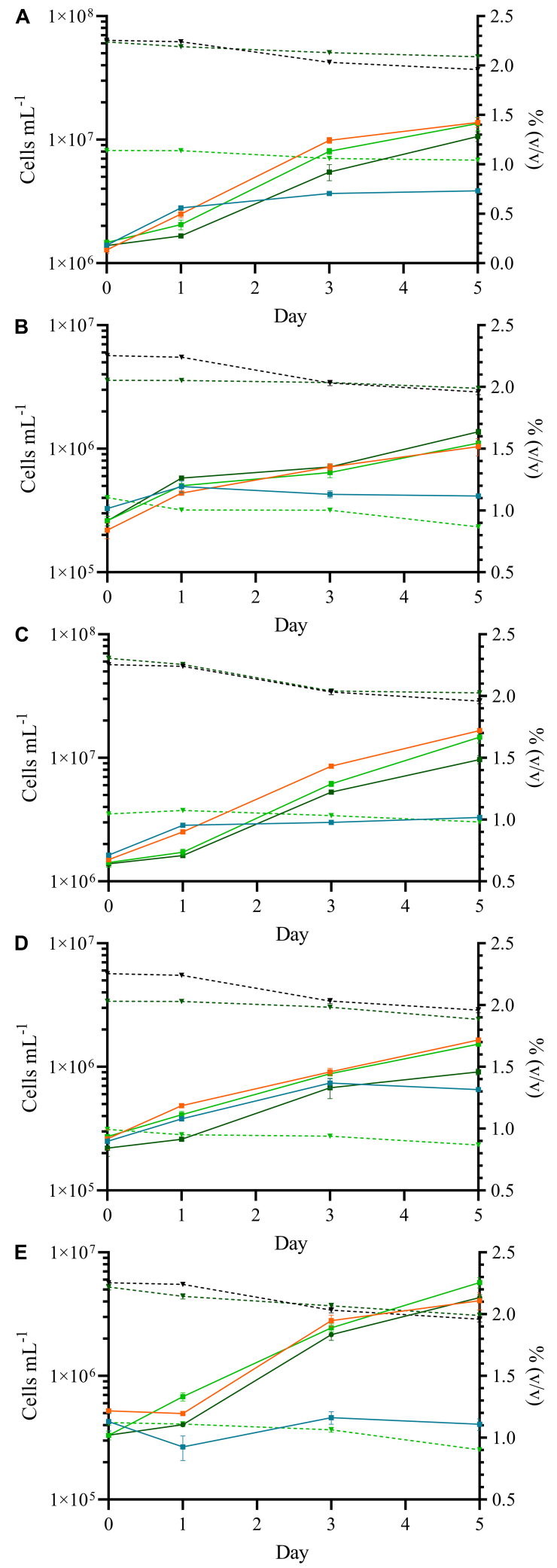

FIGURE 3 | Growth curves of Chlorella vulgaris (13-1) (A), Chlorococcum sp. (MC-1) (B), Coelastrella sp. (3-4) (C), Desmodesmus sp. (2-6) (D) and

(Continued)
FIGURE 3 | Scotiellopsis reticulata (UFA-2) (E) under mixotrophic growth in the presence of methanol. The presence of two different concentrations of methanol [3\% (v/v) (light green) and 6\% (v/v) (dark green)] were compared to a control lacking the carbon source in the medium (photoautotroph, orange) and a negative control lacking nitrogen in the medium (no algal growth, light blue). The concentration of methanol in the medium (dashed gray line, in\% $\mathrm{v} / \mathrm{v}$ ) during the time course of the experiment and in the negative control lacking algae (dashed black line) are shown on the right $y$-axis.

concentrations of glycerol (above $10 \mathrm{~g} \mathrm{~L}^{-1}$ ) (Liang et al., 2009; Lin and $\mathrm{Wu}, 2015)$.

In addition, Desmodesmus sp. (2-6) (Figure 1D) tolerated high concentrations of glycerol, its growth was comparably good with no significant difference toward the control $(p=0.241)$.

The two strains of the Scenedesmaceae family, Coelastrella sp. (3-4) (Figure 1C) and Scotiellopsis reticulata (UFA-2) (Figure 1E), grew significantly worse in the presence of $20 \mathrm{~g}$ $\mathrm{L}^{-1}$ glycerol $(p=0.002$ and $p=0.012)$. Since these two strains are highly related based on their phylogenetic trees (Minyuk et al., 2017), the presence of glycerol affects the algae most likely in a similar way. Glycerol can be produced as a response to extracellular osmotic pressure and usually is excreted by freshwater species (León and Galván, 1994; Skjånes et al., 2013). Since external glycerol can easily penetrate the cell walls of many freshwater strains it might accumulate within the cells affecting the metabolism negatively (Skjånes et al., 2013). High glycerol concentrations in the medium might prevent its excretion causing reduced growth.

The maximum quantum yield of Photosystem II (QY) correlated with the data received of the growth curves for all strains. The QY values of $0.76 \pm 0.01(p \geq 0.05)$ were stable, no significant differences between the control and growth in the presence of glycerol were observed. However, Coelastrella sp. (3-4) and Scotelliopsis reticulata (UFA-2) grew slower in the presence of $20 \mathrm{~L}^{-1}$ glycerol and their QY was decreased $(0.70 \pm 0.01, p=0.035$, and 0.015).

Interestingly, Chlorococcum sp. (MC-1) (Figure 1B), was the only strain that was able to grow significantly better in presence of $20 \mathrm{~g} \mathrm{~L}^{-1}$ of glycerol $(p=0.001)$ and took up glycerol from the medium; the glycerol concentration significantly decreased $(p=0.020)$. This strain therefore has high potential to be grown in glycerol-rich wastewater for biomass production, but specific screenings are needed to select the optimal growth conditions and concentrations.

In addition to cell counting an automated measurement of the mean cell diameter was performed. Compared to control growth conditions no significant difference of the cell diameter was observed on day 5 when grown in the presence of $2.3 \mathrm{~g} \mathrm{~L}^{-1}$ glycerol $(p \geq 0.05)$. However, comparing the cell diameters of each individual strain on day 0 and day 5 Scotiellopsis reticulata (UFA-2) showed no significant difference ( $p=0.075$ ), while Chlorococcum sp. (MC1) increased in size significantly $(p=0.018)$ and the cells of the other strain diminished in size in the presence of low concentrations of glycerol. In presence of $20 \mathrm{~g} \mathrm{~L}^{-1}$ glycerol only Coelastrella sp. (3-4) and Scotiellopsis reticulata (UFA-2) showed a significant 


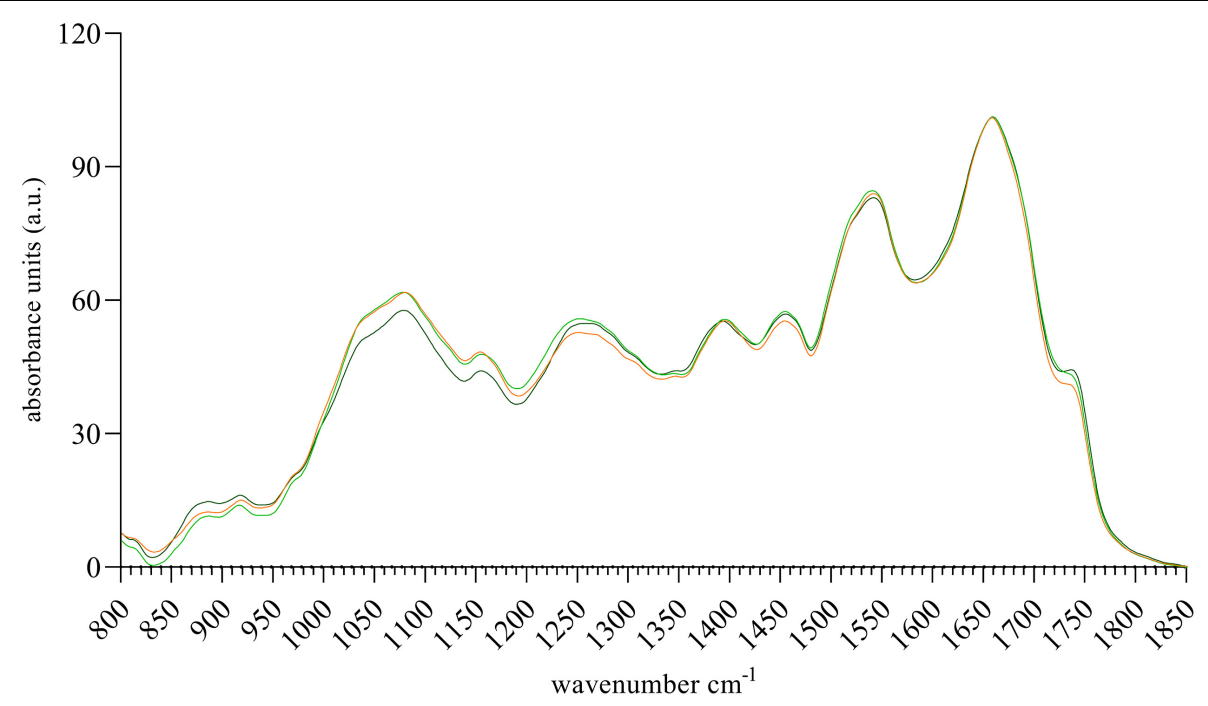

FIGURE 4 | FTIR spectra of biomass derived from Scotiellopsis reticulata (UFA-2) harvested after growth for 5 days in the presence of methanol (1.5\% (V/v), light green or $3.0 \%$ (v/v), dark green). The control culture (orange) was grown photoautotrophically in the absence of external carbon source.

smaller cell diameter ( $p=0.001$ and 0.007$)$, when compared to the control.

Fourier transform infrared spectroscopy analyses were performed on freeze-dried microalgal biomass to evaluate the effect of mixotrophic growth, 2nd derivative spectra were analyzed to make even small changes visible. These spectra allow further conclusions on the changes of specific compounds of the biomass. Comparing the FTIR spectra recorded on algal biomass produced in the presence of glycerol (mixotrophic growth) with the control lacking carbohydrates in the medium (photoautotrophic growth) (Figure 2 and Supplementary Figure 1) several changes, especially in the range of 800 to $1,200 \mathrm{~cm}^{-1}$, were observed. This region commonly is assigned to the carbohydrate fraction, it arises due to sugar ring breathing motions and C-O-C linkages (Bellamy, 1964; Nzayisenga et al., 2018). While some of the observed changes originated from glycerol itself, the reference spectrum showed corresponding peaks at e.g., $\sim 920 \mathrm{~cm}^{-1}$ (-OH group) or $\sim 1240 \mathrm{~cm}^{-1}$ (C-O stretching or -O-CH2-C-) (Kachel-Jakubowska et al., 2017), other peaks e.g., at around $870 \mathrm{~cm}^{-1}$ or $1000 \mathrm{~cm}^{-1}$ arose due to utilization of glycerol and its incorporation into the biomass. The reader should note that the spectrum of biomass derived from Chlorococcum sp. (MC-1) grown on glycerol lacked increasing peaks at $1320 \mathrm{~cm}^{-1}, 1410 \mathrm{~cm}^{-1}$ or $1650 \mathrm{~cm}^{-1}$, which are typical bands arising of the stretching vibrations of the $\delta(\mathrm{CH})$ or $C=\mathrm{C}$ vibrations in glycerol. This suggests that the provided glycerol might have been consumed and converted into algal carbohydrates. Further studies are needed, but algal biomass rich in carbohydrates presents a cheap feedstock for the production of e.g., polyhydroxyalkanoates (PHAs) either as substrate for halophilic bacteria or directly by the microalgae (Cesário et al., 2018; Costa et al., 2018).

\section{Methanol Can Support the Growth of Nordic Microalgae}

Methanol is a pollutant that appears in several different industrial wastewaters, e.g., during fiber production or from olive mills. It can also be found in concentrations up to $24.5 \mathrm{~g} \mathrm{~L}^{-1}$ in waste streams of kraft pulping mills (Lin et al., 2008). Increased growth as well as a higher lipid and amino acid accumulation of various microalgae has been observed when exposed to methanol (Nonomura and Benson, 1992; Kotzabasis et al., 1999; Theodoridou et al., 2002) at concentrations between 0.05 and $0.5 \%$ (v/v) methanol. Botryococcus braunii even was successfully exposed to 3\% (v/v) methanol (Nonomura and Benson, 1992; Theodoridou et al., 2002; Stepanov and Zolotareva, 2015). In this study two different methanol concentrations $[1.5 \%(\mathrm{v} / \mathrm{v})$ or $3.0 \%(\mathrm{v} / \mathrm{v})]$ were applied to the five Nordic microalgal strains under mixotrophic growth. Contrary to the previous studies, four of the Nordic microalgal strains exhibited either similar Chlorella vulgaris (13-1) and Chlorococcum sp. (MC-1) (Figures 3A,B) or significantly reduced growth, i.e., Coelastrella sp. (3.4) (Figure 3C, dark green curve; $p=0.0021$ ) and Desmodesmus sp. (2-6) (Figure 3D, dark green curve, $p=0.043$ ). Only Scotelliopsis reticulata (UFA-2) (see Figure 3E) showed a significantly $(p=0.0472)$ better growth in mixotrophic conditions within the 5-day time course compared to the photoautotrophic control. HPLC analysis of this culture showed a significant decrease of external methanol after 5 days compared to day 0 ( $p=0.038$ ), while the concentration of methanol in the control lacking algae not significantly $(p=0.151)$ changed. Similar to the experiment with glycerol, the maximum quantum yield of Photosystem II (QY) correlated to the growth curves for all strains. No significant difference was observed between presence of methanol and the control $(p \geq 0.05)$, although Chlorella vulgaris (13-1), Coelastrella sp. (3-4) and Desmodesmus sp. (2-6) were growing slightly slower in presence of $3 \%(\mathrm{v} / \mathrm{v})$ methanol. 

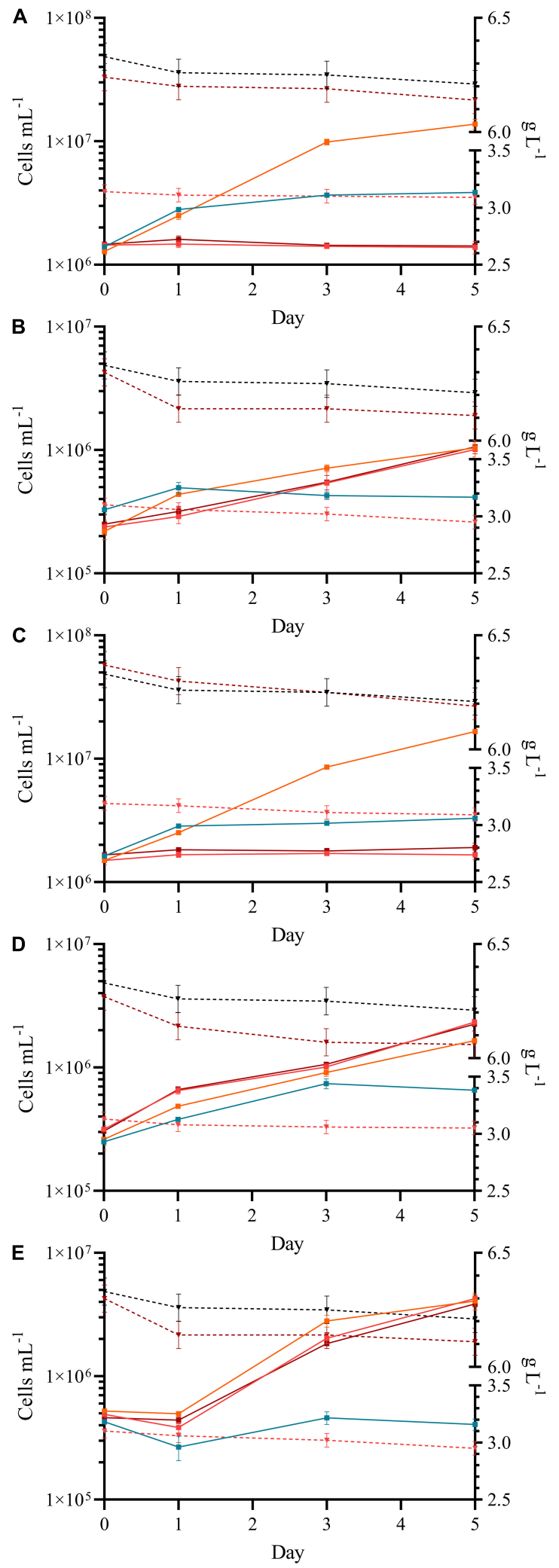

FIGURE 5 | Growth curves of Chlorella vulgaris (13-1) (A), Chlorococcum sp. (MC-1) (B), Coelastrella sp. (3-4) (C), Desmodesmus sp. (2-6) (D) and

(Continued)
FIGURE 5 | Scotiellopsis reticulata (UFA-2) (E) under mixotrophic growth in the presence of xylose. The presence of two different concentrations of xylose [ $3 \mathrm{~g} \mathrm{~L}^{-1}$ (light red) and $6 \mathrm{~g} \mathrm{~L}^{-1}$ (dark red)] were compared to a control lacking the carbon source in the medium (photoautotrophic, orange) and a negative control lacking nitrogen in the medium (no algal growth, light blue). The concentration of xylose in the medium (dashed gray line, in $\mathrm{g} \mathrm{L}^{-1}$ ) during the time course of the experiment and in the negative control lacking algae (dashed black line) are shown on the right $y$-axis.

Fourier transform infrared spectroscopy spectra as well as the 2nd derivative spectra of the microalgal biomass (see Figure 4 and Supplementary Figure 2) showed no specific difference between the photoautotrophic control (orange) and the cultures grown in the presence of different methanol concentrations. Although $1.5 \%(\mathrm{v} / \mathrm{v})$ methanol was enhancing the growth of Scotelliopsis reticulata (UFA-2) the composition of its biomass did not differ compared to the control after 5 days of growth.

Although no differences were observed in the FTIR spectra, the cell size was influenced by the presence of methanol. The cells of Chlorococcum sp. (MC1) increased in size from day 0 to day 5 ( $p=0.016$ and 0.024$)$, while the cell diameter of Scotiellopsis reticulata (UFA-2) decreased significantly ( $p=0.002$ and 0.005$)$. The cell diameter of the other strains showed no significant difference in the presence of $1.5 \%(\mathrm{v} / \mathrm{v})$ methanol $(p \geq 0.05)$, but in the presence of $3 \%(\mathrm{v} / \mathrm{v})$ methanol the cell diameter of Chlorella vulgaris $(13-1)\left(p=3.42 \mathrm{e}^{-5}\right)$ decreased while the cells of Coelastrella sp. (3-4) where swelling $(p=0.002)$. Neither of the two strains benefited from the supplementation of $3 \%(\mathrm{v} / \mathrm{v})$ methanol. Methanol enters the cells via passive transport (Cooper, 2000), however, its effect on the morphology of microalgae has not been investigated yet.

The observed difference to previous studies is explained by the applied growth conditions in those studies. Chlorella sp., for example, was grown for 45 days at a $12 \mathrm{~h}$ day/night cycle (Choi et al., 2011), but only 1\% (v/v) of methanol was added to the culture medium. Additional $\mathrm{CO}_{2}$ was fed to the algae promoting photoautotrophic growth at a much higher light intensity compared to our study. It also is worth noting that the data of the first 5 days of the study presented by Choi and coworkers (Choi et al., 2011) are very similar to our data; therefore even our Nordic Coelastrella sp. (3.4) and Desmodesmus sp. (2-6) strains might tolerate concentrations up to $3 \%(\mathrm{v} / \mathrm{v})$ of methanol when grown in a longer time interval.

\section{Nordic Microalgae Tolerate the Presence of Xylose and Show an Increased Growth}

The need for renewable energy and green biofuels requires new approaches and strategies. To be able to compete with traditional energy sources or materials algal farming must become cheaper and therefore new resources and new processes must be acquired and developed. The pulp and paper industry produces enormous amounts of wastewater containing accessible carbon that could be utilized by microalgae, xylose, a monosaccharide derived from xylan, being one of the major compounds. Nordic microalgae were therefore grown in the presence of xylose at concentrations of $3 \mathrm{~g} \mathrm{~L}^{-1}$ or $6 \mathrm{~g} \mathrm{~L}^{-1}$. Chlorella vulgaris (13-1) and Coelastrella 

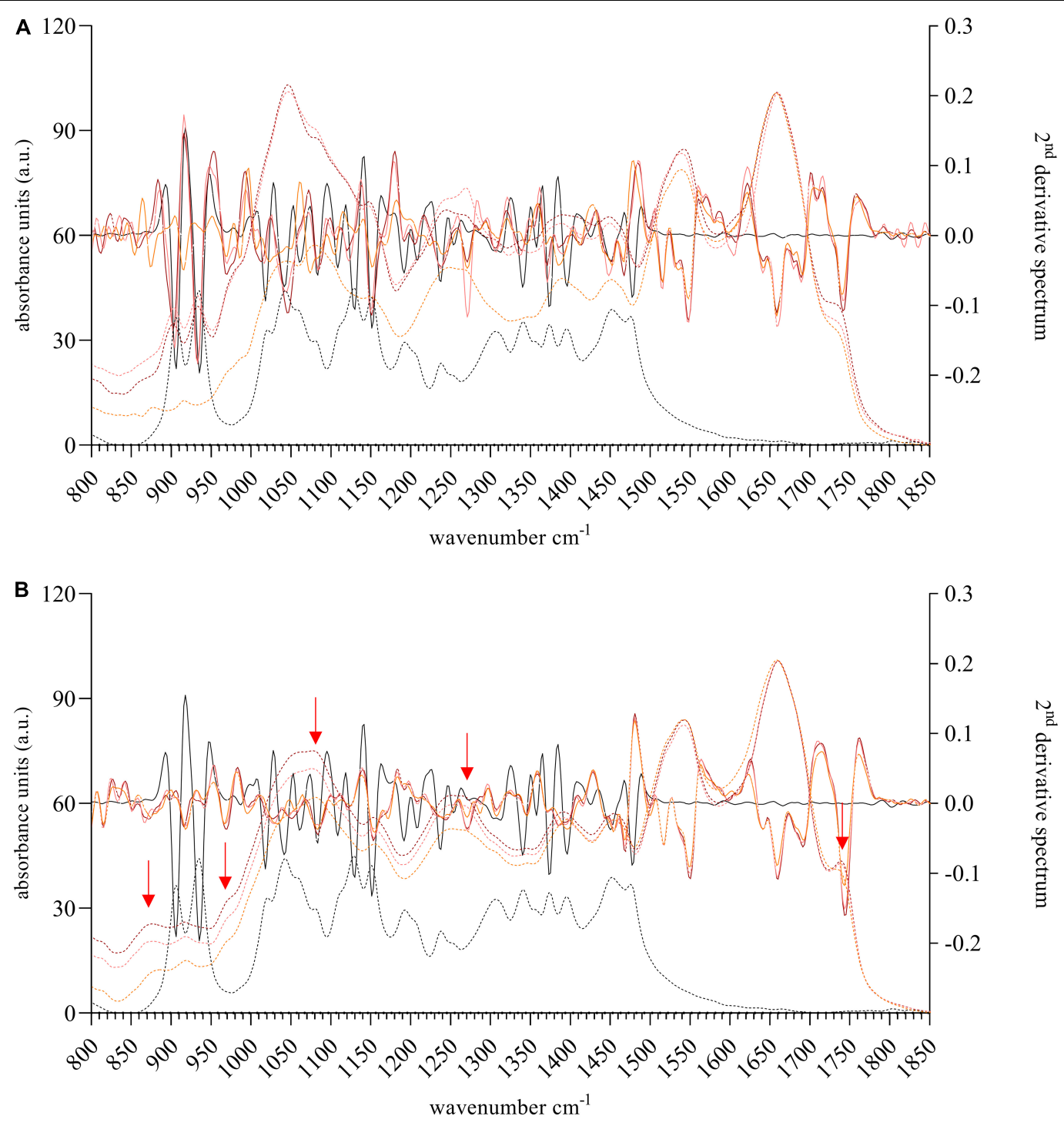

FIGURE 6 | FTIR spectra (dashed line) and 2nd derivative spectrum (solid line) of biomass derived from Coelastrella sp. (3-4) (A) and Scotiellopsis reticulata (UFA-2) (B) harvested after growth for 5 days in the presence of xylose $\left[3 \mathrm{~g} \mathrm{~L}^{-1}\right.$ (light red) or $6 \mathrm{~g} \mathrm{~L}^{-1}$ (dark red)]. The photoautotrophic control culture (orange) was grown in the absence of external carbon source, shown are also the negative control culture ( $\mathrm{N}$-free medium preventing algal growth, light blue) and the reference spectrum of xylose (black). The red arrows highlight the most prominent changes in the 2nd derivative spectra compared to the control. These cannot be assigned to xylose.

sp. (3-4) were not able to grow in the presence of xylose at these concentrations (Figures $\mathbf{5 A}, \mathbf{C}$ ). Addition of xylose immediately arrested the cell number of the culture, while the photoautotrophic control culture continued to grow. Analysis of the FTIR spectra on algal biomass of Coelastrella sp. (34) (Figure 6A) revealed the huge impact of xylose on this strain. Especially within the carbohydrate fraction multiple changes were induced by the presence of xylose (e.g., $904 \mathrm{~cm}^{-1}$, $933 \mathrm{~cm}^{-1}, 1043 \mathrm{~cm}^{-1}$ and $1238 \mathrm{~cm}^{-1}$ ). Those peaks were also dominant in the spectra received on biomass of Chlorella vulgaris (13-1) (see Supplementary Figure 3B). Three Canadian Chlorella vulgaris strains exposed to $30 \mathrm{mM}$ xylose $\left(\sim 2.7 \mathrm{~g} \mathrm{~L}^{-1}\right)$, however, accumulated lipids rapidly within $6 \mathrm{~h}$ to $12 \mathrm{~h}$ under mixotrophic conditions (Leite et al., 2016). But even the algae in these cultures decreased their amount of chlorophyll and decreased in size after $24 \mathrm{~h}$. The authors suggest that xylose might be a lipid enhancer in relatively small amounts (Leite et al., 2016). In contrast, Chlorella minutissima was able to grow on a mixture of xylose and arabinose and therefore is a good candidate to convert lignocellulosic residues for biofuel production (Freitas et al., 2021).

Quantum yields of the microalgal strains correlated with their growth performance, which was most visible by the low QY values of Chlorella vulgaris (13-1) and Coelastrella sp. (3-4) in the presence of xylose $(p=0.001$ and 0.002 , respectively $p=0.0009$ and 0.002 ), as these strains were not able to grow in its presence.

Addition of xylose also had significant impact on the algal cell diameter of those strains not able to tolerate the carbohydrate. 
The cell diameter of Chlorella vulgaris (13-1) and Coelastrella sp. (3-4) increased significantly after addition of xylose, independent of its concentration $\left(3 \mathrm{~g} \mathrm{~L}^{-1}\right.$ or $6 \mathrm{~g} \mathrm{~L}^{-1}$ xylose; $p=0.027$ and 0.006 , respectively 0.002 and 0.005 ). The increased cell diameter might arise by increased water transfer into the cells, to compensate osmotic stress. The cell diameter of Scotelliopsis reticulata (UFA-2) did not change from day 0 to day 5 ( $p=0.521$ and 0.079). While the cell diameter of Desmodemus sp. (2-6) was diminishing only in presence of $6 \mathrm{~g} \mathrm{~L}^{-1}$ xylose, the cell diameter of Chlorococcum sp. (MC1) was significantly increasing $(p=0.002$ and 0.049 ). Both strains were able to grow in the presence of xylose, independent on its concentration.

Contrary to Coelastrella sp. (3-4), its close relative Scotiellopsis reticulata (UFA-2) (Figure 5E) (Minyuk et al., 2017; Ferro et al., 2018) tolerated xylose at both concentrations and took up this carbohydrate from the medium ( $3 \mathrm{~g} \mathrm{~L}^{-1}, p=0.013$ and $\left.6 \mathrm{~g} \mathrm{~L}^{-1}, p=0.025\right)$. Desmodesmus sp. (2-6) was growing mixotrophically in the presence of xylose significantly better than the photoautotrophic control $(p=0.004$ and $p=0.011)$ (Figure 5D). At the same time a significant decrease of the xylose concentration in the medium $(p=0.04$ and $p=0.001)$ was observed. FTIR spectra of the biomass of Desmodesmus sp. (2-6) (Supplementary Figure 3A) and Scotiellopsis reticulata (UFA-2) (Figure 6B) after normalization to the amide I band indicated an increase of the carbohydrate fraction (changes are highlighted with red arrows), which is different to a simple accumulation of xylose itself. Nordic microalgae therefore can utilize xylose as a carbon source under mixotrophic conditions. Scenedesmus strains were previously shown to be superior to Chlorellaceae in utilizing xylose (Leite et al., 2016; Song and Pei, 2018). According to Song and Pei (2018) highest biomass and lipid productivity was achieved at a xylose concentration of $4 \mathrm{~g}$ $\mathrm{L}^{-1}$. While the Nordic Desmodesmus sp. (2-6) strain was able to use the carbon source immediately (no lag in growth was observed), Yang and coworkers observed a prolonged lag phase of 4 days in a Chinese Scenedesmus obliquus strain, when grown in the presence of xylose at a concentration of $4 \mathrm{~g} \mathrm{~L}^{-1}$. A xylose concentration of $6 \mathrm{~g} \mathrm{~L}^{-1}$ extended the lag phase to almost 20 days (Yang et al., 2014). Little is known about the utilization of xylose by strains of the Chlorococcum family (Yang et al., 2014). Wu and coworkers investigated growth of Chlorococcum echinozygotum in the presence of xylose and concluded that xylulose acts as a algal growth inhibitor (Wu et al., 1968). Our Nordic Chlorococcum sp. (MC-1) (Figure 5B) grew as good as the control ( $p=0.931$ and $p=0.541$, respectively, measured on day 5) although it exhibited a lag phase of 3 days. Interestingly, the xylose concentration in the medium was significantly lower at the end of the experiment ( $p=0.046$ and $p=0.023$ ).

\section{CONCLUSION}

Five Nordic microalgae were investigated regarding their ability to grow in the presence of different carbon sources and the utilization of those substrates for enhanced growth. All strains grew in the presence of $2.3 \mathrm{~g} \mathrm{~L}^{-1}$ glycerol, but only one strain, Chlorella vulgaris (13-1), showed increased growth compared to the control. Chlorococcum sp. (MC-1) was able to benefit from a much higher concentration of glycerol and increased growth when supplied with $20 \mathrm{~g} \mathrm{~L}^{-1}$ of glycerol. Contrary to culture collection strains, none of the Nordic strains took advantage from the addition of methanol to the medium within the time frame of the experiment. Supplementation with xylose indicated the need for proper screening of algal strains: While growth of Chlorella vulgaris (13-1) and Coelastrella sp. (3-4) was inhibited, Chlorococcum sp. (MC-1) and Scotiellopsis reticulata (UFA-2) grew comparable to the control, and Desmodesmus sp. (2-6) even better than the photoautotrophic control. Nordic microalgal strains therefore have a high potential for cheap and sustainable biomass production using waste streams like pulp and paper wastewater, providing feedstock for biofuel or biopolymer production. Our results support the development of new technologies turning waste streams into resources in a circular economy concept.

\section{DATA AVAILABILITY STATEMENT}

The raw data supporting the conclusions of this article will be made available by the authors, without undue reservation.

\section{AUTHOR CONTRIBUTIONS}

CF and MP designed the experiments. MP and KS performed the experimental work, data collection, analysis, and interpretation under the supervision of CF. SS performed the HPLC analysis of the carbon sources under the supervision of LJ. CF conceptualized the project and was responsible for funding acquisition. All authors were involved in the preparation of the manuscript and approved its submission.

\section{ACKNOWLEDGMENTS}

We would like to thank the Swedish Energy Agency (Grant No. 2018-017772, project: 48007-1), Vinnova (2017-03301), the NordForsk NCoE program "NordAqua" (Project No. 82845), FORMAS (2019-00492) and Umeå University as well as the Industrial Doctoral School at Umeå University for financial support. We further would like to thank András Gorzsás from the Vibrational Spectroscopy Core Facility at Umeå University for the provided expertise and the data processing of the FTIR spectra and Zivan Gojkovic at Instituto Tecnológico de Canarias for the interesting discussions prior to this study.

\section{SUPPLEMENTARY MATERIAL}

The Supplementary Material for this article can be found online at: https://www.frontiersin.org/articles/10.3389/fmars.2022. 830800/full\#supplementary-material 


\section{REFERENCES}

Acién, F. G., Gómez-Serrano, C., Morales-Amaral, M. M., Fernández-Sevilla, J. M., and Molina-Grima, E. (2016). Wastewater treatment using microalgae: how realistic a contribution might it be to significant urban wastewater treatment? Appl. Microbiol. Biotechnol. 100, 9013-9022. doi: 10.1007/s00253-016-78 35-7

Barbosa, M. J., Hadiyanto, and Wijffels, R. H. (2004). Overcoming shear stress of microalgae cultures in sparged photobioreactors. Biotechnol. Bioeng. 85, 78-85. doi: 10.1002/bit.10862

Bellamy, L. J. (1964). The Infra-Red Spectra of Complex Molecules, 2nd Edn. New York: Methuen.

Bertani, G. (1951). Studies on lysogenesis i. J. Bacteriol. 62, 293-300. doi: 10.1128/ jb.62.3.293-300.1951

Bischoff, H. W., and Bold, H. C. (1963). Phycological studies. IV. Some Algae from Enchanted Rock and Related Algal Species. Univ Tex. Publ. 6318,1-95.

Boretti, A., and Rosa, L. (2019). Reassessing the projections of the World Water Development Report. Npj Clean Wat. 2:15. doi: 10.1038/s41545-019-0 039-9

Cesário, M. T., da Fonseca, M. M. R., Marques, M. M., and De Almeida, M. C. M. D. (2018). Marine algal carbohydrates as carbon sources for the production of biochemicals and biomaterials. Biotechnol. Adv. 36, 798-817. doi: 10.1016/j. biotechadv.2018.02.006

Choi, W.Y., Oh, S.H., Seo, Y.C., Kim, G.B., Kang, D.H., Lee, S.Y., et al. (2011). Effects of methanol on cell growth and lipid production from mixotrophic cultivation of Chlorella sp. Biotechnol. Bioprocess Eng. 16, 946-955. doi: 10. 1007/s12257-010-0394-3

Christenson, L., and Sims, R. (2011). Production and harvesting of microalgae for wastewater treatment, biofuels, and bioproducts. Biotechnol. Adv. 29, 686-702. doi: 10.1016/j.biotechadv.2011.05.015

Cooper, G. M. (2000). “Transport of small molecules," in The Cell: A Molecular Approach, 2nd Edn, ed. Sinauer Associates Inc. (Sunderland, MA: Sinauer Associates).

Costa, S. S., Miranda, A. L., Andrade, B. B., Assis, D., de, J., Souza, C. O., et al. (2018). Influence of nitrogen on growth, biomass composition, production, and properties of polyhydroxyalkanoates (PHAs) by microalgae. Int. J. Biol. Macromol. 116, 552-562. doi: 10.1016/j.ijbiomac.2018.05.064

Dobson, R., Gray, V., and Rumbold, K. (2012). Microbial utilization of crude glycerol for the production of value-added products. J. Ind. Microbiol. Biotechnol. 39, 217-226. doi: 10.1007/s10295-011-1038-0

Ferro, L., Colombo, M., Posadas, E., Funk, C., and Muñoz, R. (2019a). Elucidating the symbiotic interactions between a locally isolated microalga Chlorella vulgaris and its co-occurring bacterium Rhizobium sp. in synthetic municipal wastewater. J. Appl. Phycol 31, 2299-2310. doi: 10.1007/s10811-019-1741-1

Ferro, L., Gojkovic, Z., Muñoz, R., and Funk, C. (2019b). Growth performance and nutrient removal of a Chlorella vulgaris-Rhizobium sp. co-culture during mixotrophic feed-batch cultivation in synthetic wastewater. Algal Res. 44:101690. doi: 10.1016/j.algal.2019.101690

Ferro, L., Gentili, F. G., and Funk, C. (2018). Isolation and characterization of microalgal strains for biomass production and wastewater reclamation in Northern Sweden. Algal Res. 32, 44-53. doi: 10.1016/j.algal.2018.03.006

Freitas, B. C. B., Morais, M. G., and Costa, J. A. V. (2021). Chlorella minutissima grown with xylose and arabinose in tubular photobioreactors: Evaluation of kinetics, carbohydrate production, and protein profile. Can. J. Chem. Eng. cjce. 24116 . doi: $10.1002 /$ cjce. 24116

Gojkovic, Z., Lindberg, R. H., Tysklind, M., and Funk, C. (2019). Northern green algae have the capacity to remove active pharmaceutical ingredients. Ecotoxicol. Environ. Saf. 170, 644-656. doi: 10.1016/j.ecoenv.2018.12.032

Gorzsás, A., and Sundberg, B. (2014). Chemical fingerprinting of Arabidopsis using Fourier transform infrared (FT-IR) spectroscopic approaches. Method. Mol. Biol. 1062, 317-352.

Goswami, R. K., Mehariya, S., Verma, P., Lavecchia, R., and Zuorro, A. (2021). Microalgae-based biorefineries for sustainable resource recovery from wastewater. J. Water Process Eng. 40:101747. doi: 10.1016/j.jwpe.2020.101747

Gouveia, L. (2011). "Microalgae as a Feedstock for Biofuels", in Microalgae as a Feedstock for Biofuels SpringerBriefs in Microbiology, ed. L. Gouveia (Berlin: Springer), 1-69. doi: 10.1007/978-3-642-17997-6_1
Hena, S., Gutierrez, L., and Croué, J.-P. (2021). Removal of pharmaceutical and personal care products (PPCPs) from wastewater using microalgae: A review. J. Hazard. Mater. 403:124041. doi: 10.1016/j.jhazmat.2020.124041

Heredia-Arroyo, T., Wei, W., Ruan, R., and Hu, B. (2011). Mixotrophic cultivation of Chlorella vulgaris and its potential application for the oil accumulation from non-sugar materials. Biomass Bioenergy 35, 2245-2253. doi: 10.1016/j. biombioe.2011.02.036

Kachel-Jakubowska, M., Matwijczuk, A., and Gagoś, M. (2017). Analysis of the physicochemical properties of post-manufacturing waste derived from production of methyl esters from rapeseed oil. Int. Agrophysics 31, 175-182. doi: 10.1515/intag-2016-0042

Kong, W.-B., Yang, H., Cao, Y.-T., Song, H., Hua, S.-F., and Xia, C.-G. (2013). Effect of Glycerol and Glucose on the Enhancement of Biomass, Lipid and Soluble Carbohydrate Production by Chlorella vulgaris in Mixotrophic Culture. Food Technol. Biotechnol. 51, 62-69.

Kotzabasis, K., Hatziathanasiou, A., Bengoa-Ruigomez, M. V., Kentouri, M., and Divanach, P. (1999). "Methanol as alternative carbon source for quicker efficient production of the microalgae Chlorella minutissima: Role of the concentration and frequence of administration," in Progress in Industrial Microbiology Marine Bioprocess Engineering, eds R. Osinga, J. Tramper, J. G. Burgess, and R. H. Wijffels (Amsterdam: Elsevier), 357-362. doi: 10.1016/S0079-6352(99)80128-3

Leite, G. B., Paranjape, K., Abdelaziz, A. E. M., and Hallenbeck, P. C. (2015). Utilization of biodiesel-derived glycerol or xylose for increased growth and lipid production by indigenous microalgae. Bioresour. Technol. 184, 123-130. doi: 10.1016/j.biortech.2014.10.117

Leite, G. B., Paranjape, K., and Hallenbeck, P. C. (2016). Breakfast of champions: Fast lipid accumulation by cultures of Chlorella and Scenedesmus induced by xylose. Algal Res. 16, 338-348. doi: 10.1016/j.algal.2016.03.041

León, R., and Galván, F. (1994). Halotolerance studies on Chlamydomonas reinhardtii: glycerol excretion by free and immobilized cells. J. Appl. Phycol. 6, 13-20. doi: 10.1007/BF02185898

Liang, Y., Sarkany, N., and Cui, Y. (2009). Biomass and lipid productivities of Chlorella vulgaris under autotrophic, heterotrophic and mixotrophic growth conditions. Biotechnol. Lett. 31, 1043-1049. doi: 10.1007/s10529-009-9975-7

Lin, T.-S., and Wu, J.-Y. (2015). Effect of carbon sources on growth and lipid accumulation of newly isolated microalgae cultured under mixotrophic condition. Bioresour. Technol. 184, 100-107. doi: 10.1016/j.biortech.2014.11. 005

Lin, Y., He, Y., Meng, Z., and Yang, S. (2008). Anaerobic treatment of wastewater containing methanol in upflow anaerobic sludge bed (UASB) reactor. Front. Environ. Sci. Eng. China 2:241-246. doi: 10.1007/s11783-008-0040-x

Mehariya, S., Goswami, R. K., Karthikeysan, O. P., and Verma, P. (2021). Microalgae for high-value products: A way towards green nutraceutical and pharmaceutical compounds. Chemosphere 280:130553. doi: 10.1016/j. chemosphere.2021.130553

Minyuk, G., Chelebieva, E., Chubchikova, I., Dantsyuk, N., Drobetskaya, I., Sakhon, E., et al. (2017). Stress-induced secondary carotenogenesis in Coelastrella rubescens (Scenedesmaceae, Chlorophyta), a producer of valueadded keto-carotenoids. ALGAE 32, 245-259. doi: 10.4490/algae.2017.32.8.6

Molinuevo-Salces, B., Riaño, B., Hernández, D., and Cruz García-González, M. (2019). "Microalgae and Wastewater Treatment: Advantages and Disadvantages," in Microalgae Biotechnology for Development of Biofuel and Wastewater Treatment, eds M. D. A. Alam and Z. Wang (Singapore: Springer), 505-533. doi: 10.1007/978-981-13-2264-8_20

Munthe, J., Eriksson, E., Ek, M., Öman, A., Karlsson, P.-E., and Stål, J. (2011). Water Profile for the Swedish forest industry. Sweden: IVL Svenska Miljöinstitutet.

Nonomura, A. M., and Benson, A. A. (1992). The path of carbon in photosynthesis: improved crop yields with methanol. Proc. Natl. Acad. Sci. 89, 9794-9798. doi: 10.1073 /pnas.89.20.9794

Nzayisenga, J. C., Eriksson, K., and Sellstedt, A. (2018). Mixotrophic and heterotrophic production of lipids and carbohydrates by a locally isolated microalga using wastewater as a growth medium. Bioresour. Technol. 257, 260-265. doi: 10.1016/j.biortech.2018.02.085

Onen Cinar, S., Chong, Z. K., Kucuker, M. A., Wieczorek, N., Cengiz, U., and Kuchta, K. (2020). Bioplastic Production from Microalgae: A Review. Int. J. Environ. Res. Public. Health 17:3842. doi: 10.3390/ijerph17113842 
Plöhn, M., Spain, O., Sirin, S., Silva, M., Escudero-Oñate, C., Ferrando-Climent, L., et al. (2021). Wastewater treatment by microalgae. Physiol. Plant. 173, 568-578. doi: $10.1111 /$ ppl.13427

Rai, M. P., Nigam, S., and Sharma, R. (2013). Response of growth and fatty acid compositions of Chlorella pyrenoidosa under mixotrophic cultivation with acetate and glycerol for bioenergy application. Biomass Bioenergy 58, 251-257. doi: 10.1016/j.biombioe.2013.08.038

Rolton, A., McCullough, A., Tuckey, N. P. L., Finnie, B., Cooper, I., Packer, M. A., et al. (2020). Early biomarker indicators of health in two commercially produced microalgal species important for aquaculture. Aquaculture 521:735053. doi: 10.1016/j.aquaculture.2020.735053

Sivaramakrishnan, R., and Incharoensakdi, A. (2018). Utilization of microalgae feedstock for concomitant production of bioethanol and biodiesel. Fuel 217, 458-466. doi: 10.1016/j.fuel.2017.12.119

Skjånes, K., Rebours, C., and Lindblad, P. (2013). Potential for green microalgae to produce hydrogen, pharmaceuticals and other high value products in a combined process. Crit. Rev. Biotechnol. 33, 172-215. doi: 10.3109/07388551. 2012.681625

Sluiter, A., Hames, B., Ruiz, R., Scarlata, C., Sluiter, J., and Templeton, D. (2008). Determination of Sugars, Byproducts, and Degradation Products in Liquid Fraction Process Samples: Laboratory Analytical Procedure (LAP). United States: National Renewable Energy Laboratory, 14. Issue Date: 12/08/2006. Tech. Rep.

Song, M., and Pei, H. (2018). The growth and lipid accumulation of Scenedesmus quadricauda during batch mixotrophic/heterotrophic cultivation using xylose as a carbon source. Bioresour. Technol. 263, 525-531. doi: 10.1016/j.biortech. 2018.05.020

Spain, O., Plöhn, M., and Funk, C. (2021). The cell wall of green microalgae and its role in heavy metal removal. Physiol. Plant 173, 1-10.

Stepanov, S. S., and Zolotareva, E. K. (2015). Methanol-induced stimulation of growth, intracellular amino acids, and protein content in Chlamydomonas reinhardtii. J. Appl. Phycol. 27, 1509-1516. doi: 10.1007/s10811-014-0 445-9
Suresh Kumar, K., Dahms, H.-U., Won, E.-J., Lee, J.-S., and Shin, K.-H. (2015). Microalgae - A promising tool for heavy metal remediation. Ecotoxicol. Environ. Saf. 113, 329-352. doi: 10.1016/j.ecoenv.2014.12.019

Theodoridou, A., Dörnemann, D., and Kotzabasis, K. (2002). Light-dependent induction of strongly increased microalgal growth by methanol. Biochim. Biophys. Acta BBA Gen. Subj. 1573, 189-198. doi: 10.1016/S0304-4165(02) 00438-5

Wu, M., Alston, R. E., and Mabry, T. J. (1968). Xylulose, an algal growth inhibitor ${ }^{2}$. J. Phycol. 4, 206-211. doi: 10.1111/j.1529-8817.1968.tb04716.x

Yang, F., Hanna, M. A., and Sun, R. (2012). Value-added uses for crude glycerol-a byproduct of biodiesel production. Biotechnol. Biofuels 5, 13. doi: 10.1186/17546834-5-13

Yang, S., Liu, G., Meng, Y., Wang, P., Zhou, S., and Shang, H. (2014). Utilization of xylose as a carbon source for mixotrophic growth of Scenedesmus obliquus. Bioresour. Technol. 172, 180-185. doi: 10.1016/j.biortech.2014.08.122

Conflict of Interest: The authors declare that the research was conducted in the absence of any commercial or financial relationships that could be construed as a potential conflict of interest.

Publisher's Note: All claims expressed in this article are solely those of the authors and do not necessarily represent those of their affiliated organizations, or those of the publisher, the editors and the reviewers. Any product that may be evaluated in this article, or claim that may be made by its manufacturer, is not guaranteed or endorsed by the publisher.

Copyright (c) 2022 Plöhn, Scherer, Stagge, Jönsson and Funk. This is an open-access article distributed under the terms of the Creative Commons Attribution License (CC BY). The use, distribution or reproduction in other forums is permitted, provided the original author(s) and the copyright owner(s) are credited and that the original publication in this journal is cited, in accordance with accepted academic practice. No use, distribution or reproduction is permitted which does not comply with these terms. 